\title{
PERSPECTIVES
}

\section{Prediction of antibiotic resistance: time for a new preclinical paradigm?}

\author{
Morten O. A. Sommer, Christian Munck, Rasmus Vendler Toft-Kehler \\ and Dan I. Andersson
}

Abstract | Predicting the future is difficult, especially for evolutionary processes that are influenced by numerous unknown factors. Still, this is what is required of drug developers when they assess the risk of resistance arising against a new antibiotic candidate during preclinical development. In this Opinion article, we argue that the traditional procedures that are used for the prediction of antibiotic resistance today could be markedly improved by including a broader analysis of bacterial fitness, infection dynamics, horizontal gene transfer and other factors. This will lead to more informed preclinical decisions for continuing or discontinuing the development of drug candidates.

Antibiotic resistance is increasingly threatening our ability to treat bacterial infections and to carry out various medical treatments and procedures, including chemotherapy, transplants and surgery, which require effective antibiotics to reduce complications. The emergence of antibiotic-resistant bacteria limits the clinical use of antibiotics and, as resistant bacteria become more prevalent, there is an increasing concern that existing antibiotics will become ineffective against these pathogens ${ }^{1}$. Fortunately, awareness is increasing, and actions to tackle antibiotic resistance and remedy the funding gaps and market inefficiencies are discussed at international levels. In addition, initiatives are being taken to improve diagnostics and to promote the development of new antibiotics that have a more extended clinical lifetime ${ }^{1}$.

During the preclinical evaluation of new antibiotic candidates, the development of resistance is assessed in vitro with the aim of early detection and termination of drug candidates with which resistance develops too quickly. However, research into the specific factors that drive the evolution of antibiotic resistance in the laboratory and in the clinical setting has not been adequately incorporated into preclinical drug-testing programmes. Consequently, drug candidates that have the potential to be successful in the clinic may be wrongly dismissed, or antibiotics that may be rendered useless by the rapid acquisition of resistance genes could proceed through the drug development process.

In this Opinion article, we describe the various factors that influence the evolution of antibiotic resistance and discuss these factors in the context of early drug discovery, with the aim of encouraging an updated paradigm for assessing the evolution of resistance during the development of new antibiotics. We propose that the use of in vitro mutation rates as a predictor of the evolution of resistance should be replaced by a broader analysis of the major factors that can influence the evolution of resistance, including bacterial fitness (that is, the relative ability to survive, reproduce and propagate in an environment), infection dynamics, cross-resistance (that is, when resistance to one drug confers resistance to other drugs), co-selection and horizontal gene transfer (HGT). This updated preclinical paradigm challenges established procedures and, by using additional risk-assessment criteria, it may provide better predictions to determine whether a drug candidate should advance to clinical development. In particular, we propose that drug candidates that show a high rate of formation of resistant mutants should not be discarded too early in drug development and that an evaluation of the potential for antibiotic resistance to be acquired through HGT should be included in early preclinical analysis.

\section{Evolution of antibiotic resistance}

Assessment of the evolution of resistance is a key step in the preclinical development of new antibiotics. Resistance can develop through two distinct paths: vertical evolution, whereby mutations that enhance antibiotic tolerance are selected for and are transmitted to the progeny (FIG. 1a); or horizontal evolution, whereby antibiotic resistance genes are acquired from other bacteria, through conjugation, transduction or transformation, and are subsequently transmitted to the progeny (FIG. 1 b). The relative contribution of each of these evolutionary paths to the development of clinical resistance is not yet fully elucidated, but the comprehensive genomic characterization of human pathogens during past decades has shown that horizontal evolution contributes to the majority of current resistance problems ${ }^{2}$.

The evolution, transmission and maintenance of antibiotic resistance in a population of bacteria are driven by the complex interplay of several factors, including the rate at which resistance genes and mutations arise, the level of resistance that is conferred by the particular resistance mechanism, the relative fitness of the resistant mutant at different drug concentrations and the strength of various selective pressures that drive the evolution of resistance (FIG. 2). In addition, epistatic interactions (in which the effect of one gene locus is influenced by the presence of one or more modifier genes), the evolution of compensatory mutations, the development of cross-resistance, and co-selection of genes that induce drug resistance or drug sensitivity with other genes, can influence the evolution of resistance. In this section, we briefly describe these processes and discuss how they contribute to the development of antibiotic resistance.

Basic factors. The rate at which resistance genes and mutations arise (also known as mutation supply rate) is determined by 
a Vertical evolution

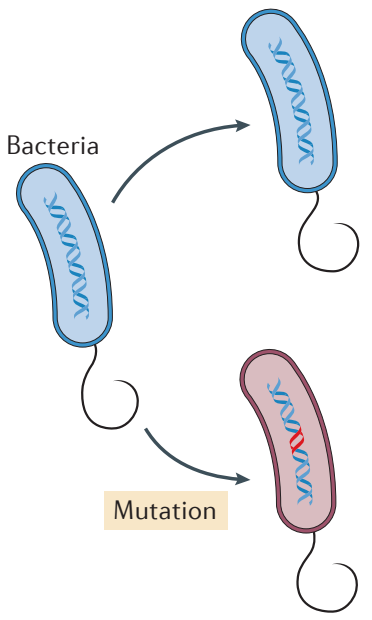

Figure 1 | Evolution of resistance. Resistance can evolve through two basic mechanisms: vertical evolution (part a) and horizontal evolution (part b). a | Vertical evolution represents the emergence of de novo mutations in the bacterial genome and their subsequent transfer to the progeny. $\mathbf{b}$ |Horizontal acquisition of resistance genes, known as horizontal gene transfer, can instead occur through phage transduction, conjugation or natural transformation. Phage transduction is the process by which a bacteriophage attaches to a recipient bacterial cell and injects its viral DNA, which is then integrated into the chromosome. Bacterial conjugation occurs through direct contact between the donor and recipient bacterial cells, and mediates the acquisition of conjugative plasmids and integrative conjugative elements. Natural transformation occurs when naked DNA is released by lysing donor cells and is subsequently taken up by the recipient bacterial cell. All of these genetic processes mediate the acquisition of mutations and/or genes (represented by red DNA tracts) that confer antibiotic resistance to the recipient bacterium. Blue cells are sensitive bacteria, whereas red cells are resistant.

population sizes, rates of mutation and HGT. The degree of genetic heterogeneity in a bacterial population in humans is largely influenced by the rate at which mutations arise and potential bottlenecks during intra-host or inter-host transmission ${ }^{3}$. Our knowledge of bacterial population sizes in infected hosts is limited but, in cases in which they are known, there generally seem to be $>10^{8}$ bacteria per infected host per infection site ${ }^{4-8}$. As spontaneous mutation rates in bacteria are typically around $10^{-10}$ per nucleotide per generation, it is estimated that resistant mutants are likely to be present in the infecting population ${ }^{9-11}$.

The level of resistance that develops can vary depending on the resistance mechanism and the conditions under which resistance is measured. Some resistance mechanisms confer resistance levels that exceed the solubility limit of the antibiotic ${ }^{12}$, whereas other resistance mechanisms result in small increases in resistance that can only be detected with sensitive 'timekill experiments' that measure the rate at which bacteria are killed during exposure to an antibiotic ${ }^{13}$. Measuring the levels of resistance is complicated by the nonlinear shape of the pharmacodynamic response curve (that is, bacterial growth as a function of drug concentration) and how bacterial growth conditions affect the measured resistance levels. For some resistance mechanisms, bacterial growth is unaffected by the use of increasing concentrations of antibiotic, whereas for other mechanisms, growth decreases progressively until the minimal inhibitory concentration (MIC) is reached ${ }^{14-18}$. A potential complication of such measurements is the presence of cases of resistance that is only induced in response to the antibiotic (also known as inducible resistance $)^{19}$. Therefore, the relative fitness of resistant bacteria might be unaffected or might vary depending on the concentration of antibiotic ${ }^{20}$. Furthermore, several recent studies have shown that resistance may be dependent on environmental conditions (for example, through collective bacterial interactions or the presence of specific metabolites or growth conditions that can modulate and alter the phenotypic expression of a specific resistance mechanism ${ }^{20,21}$ ).

As shown by theoretical work, epidemiology and laboratory experiments, the relative fitness of a drug-resistant bacterium, both in the absence and presence of the drug, is a key parameter in determining its evolutionary success. Most experimentally examined resistance mechanisms result in decreased bacterial fitness compared with a susceptible ancestor in the absence of antibiotic, which is typically analysed by measuring growth rates under different conditions ${ }^{3,21-23}$. However, there are also exceptions in which resistance seems neutral or beneficial to the fitness of the drug-resistant bacterium ${ }^{21,24}$. On the basis of the limited studies that have been carried out, it seems likely that measurements of bacterial fitness in the laboratory can be used as reasonable predictors for the evolutionary success of antibiotic-resistant bacteria in clinical settings. Indeed, the most common mutations - providing rifampicin and aminoglycoside resistance to clinical isolates of Mycobacterium tuberculosis and Staphylococcus aureus - are associated with the lowest fitness costs ${ }^{25-29}$. Furthermore, the fitness cost of a resistance mechanism might vary between species and influence the spread of resistance (for example, resistance to vancomycin). Indeed, in enterococci, the fitness cost is very low and antibiotic resistance dissemination is worldwide, whereas in S. aureus the fitness cost is high and very little spread has been observed $^{30}$. Thus, fitness costs seem to inversely correlate with successful spread in clinical settings.

Bacteria in different environments are exposed to a range of various antimicrobial agents with time-variable and space-variable concentrations and, consequently, the strength of selection in natural settings is difficult to assess. Furthermore, as shown in recent in vitro evolution experiments, the outcome of evolution varies depending on whether it occurs at drug concentrations that inhibit pathogen growth (>MIC) or that allow the growth of both susceptible and resistant bacteria $(<\mathrm{MIC})^{31}$. Thus, the rate of emergence of mutations and the type of mutants that are selected for during evolution differ between $>$ MIC (lethal) and $<$ MIC (non-lethal) selective pressures. Indeed, at high lethal antibiotic concentrations, rare mutations that provide a high level of resistance in a single genetic event have a high probability of being selected and transmitted to progeny, whereas, at non-lethal antibiotic concentrations, the selected mutants often result from an accumulation of multiple mutations that, when combined, result in a high level of resistance ${ }^{14,31-33}$. Importantly and paradoxically, the weaker the selection for resistance the stronger the enrichment for more problematic mutants that have a low fitness cost and mutator phenotypes (that is, an increased mutation rate owing to defects in DNA repair) that increase the risk of successful spread and further resistance evolution, respectively ${ }^{31}$. 
Additional key factors. Epistasis can also affect the outcome of the evolution of antibiotic resistance ${ }^{34,35}$. Epistasis makes it more challenging to predict potential antibiotic resistance phenotypes after mutation or HGT of genes into new genetic environments, and a greater understanding of the details of epistatic interactions could lead to better predictions of evolutionary outcomes and the resultant phenotypes. Epistatic interactions could affect the level of resistance or the relative fitness of a resistant mutant. For example, in vitro evolution experiments show that epistasis can decrease the fitness costs of mutations and confer resistance to different classes of antibiotics, including streptomycin, rifampicin, fusidic acid and ciprofloxacin ${ }^{36-45}$. It has been shown that epistatic interactions may constrain and direct the order in which antibiotic resistance mutations arise ${ }^{39,45-52}$. Similarly, epistasis has a role in maintaining plasmids that confer antibiotic resistance in bacteria. Typically, when a plasmid enters a naive host, it decreases its fitness; however, these costs can be reduced by subsequent genetic mutations in the plasmid or host chromosome, or a combination of both, thereby enhancing plasmid stability and persistence ${ }^{53-58}$. A specific variant of epistasis known as 'collateral sensitivity', in which the development of resistance to one antibiotic is associated with higher susceptibility to another antibiotic, can also influence resistance levels ${ }^{59-62}$. Collateral sensitivity seems to be a relatively common phenomenon; however, in most cases, the mechanisms remain poorly understood ${ }^{63-67}$.

Another complicating factor in predicting resistance evolution, which is difficult to quantify, is the co-selection of one resistance gene with another resistance gene owing to their physical linkage.

Co-selection could occur when two or more genes are present on the same chromosome, plasmid, transposon or integron. Indeed, most antibiotic resistance plasmids carry multiple antibiotic resistance genes and a consequence of this genetic linkage is that selection for one of the antibiotic genes then selects for other linked resistance genes ${ }^{68-70}$. Therefore, co-selection could decrease the effect of purifying selection (that is, selection against mutations and/or genes that are deleterious) on a resistance gene that is associated with a high fitness cost and, consequently, it could disassociate the development of resistance to a particular antibiotic from the use of that antibiotic. Such disassociation is supported by

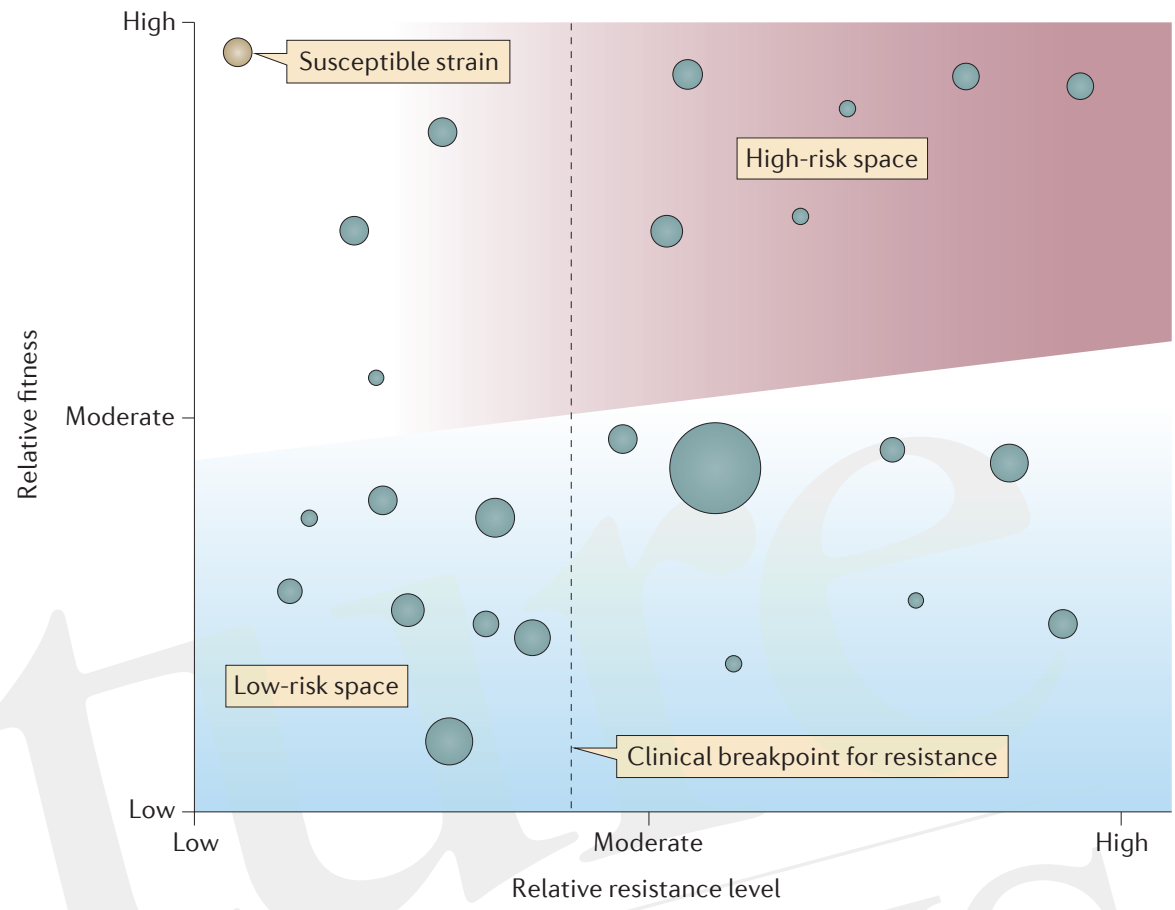

Figure 2 | Factors that determine the risk of evolution of antibiotic resistance. Each circle represents a hypothetical mutation or a horizontal gene transfer (HGT) event that leads to increased tolerance to a given antibiotic. The size of the circles represents the rate by which mutations or HGT events occur (corresponding to in vivo rate x population size). Their position on the $y$-axis reflects the relative fitness imposed by the genetic change (classified as low, medium or high), whereas the position on the $\mathrm{x}$-axis reflects the level of resistance conferred (classified as low, medium or high). The blue and red shading indicate low and high-risk spaces with regard to development of antibiotic resistance. A HGT or mutation event in the red space is more likely to get fixed in a population in the presence of antibiotic as the imposed fitness cost is relatively low and the conferred increase in tolerance is high. The vertical dashed line indicates the clinical breakpoint for resistance, which is the antibiotic concentration that defines whether a bacterial species is predicted to be treatable with the antibiotic.

intervention studies in which a reduction in the clinical use of a particular class of antibiotics did not result in a decrease in the frequency of that resistance in the community $^{71-73}$. Generally, co-selection makes prediction very challenging, as the evolutionary success of the acquisition of a resistance gene or a mutation is not only influenced by the level of resistance and the fitness it confers but also by its genetic context and any selective pressures that act on the neighbouring genes. Furthermore, cross-resistance mechanisms (for example, porin mutations or the activation of antibiotic efflux systems) that confer resistance within and between different antibiotic classes or biocides could also complicate predictions by influencing resistance levels to many different drugs. Thus, as most pathogens are exposed to multiple antibiotics, simultaneously or sequentially, bacteria that are multidrug resistant (MDR) will generally be the most successful in terms of survival and multiplication.

\section{Current assessments are limited}

In the development of new antibiotics, the current preclinical assessments of resistance largely focus on the acquisition of resistance by de novo mutations in conjunction with assessing the ability of the drug candidate to kill MDR pathogens. Studies of the evolution of antibiotic resistance are typically limited to determining the rate of acquisition of spontaneous mutations that confer resistance for a specific drug candidate and to determining the overall potential evolution of resistance through repeated exposure of a bacterial population to constant or increasing concentrations of the drug candidate. These approaches are beneficial in that they provide a well-defined readout of the potential genetic mutations that can confer resistance towards a particular drug. However, as outlined above, many other factors influence the evolution of resistance and therefore these approaches are too simplistic. We propose considering other factors that could be of greater importance in predicting future clinical resistance. 


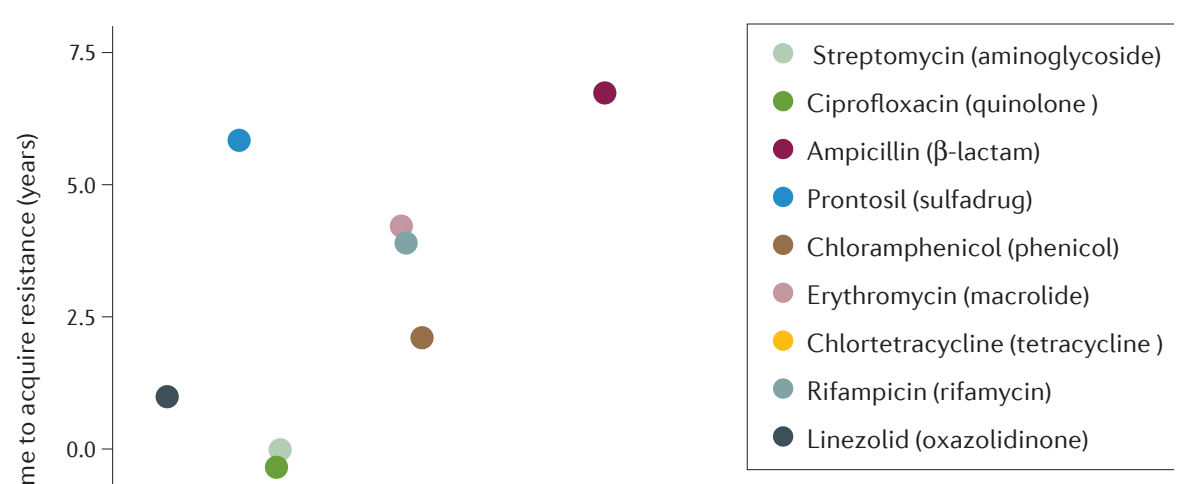

$\begin{array}{llllll}10^{-10} & 10^{-9} & 10^{-8} & 10^{-7} & 10^{-6} & 10^{-5}\end{array}$

Spontaneous resistance mutation (rate per cell per generation)

Figure 3 | The in vitro rate of resistance mutations does not correlate with the burden of resistance in the clinic. Time to first observed resistance from first use of the drug is plotted in the $y$-axis as a function of the rate of emergence of spontaneous resistance mutations shown in the $x$-axis. Time is shown in years, whereas the rate of mutation emergence is shown as the number of mutations per cell per generation. Note, that for some drugs, emergence of resistance was reported even before its clinical use. The data points are slightly jittered on both axes to avoid over-plotting. Data on mutation rate was obtained from REFS 15,16,76,90-96 and data on time to resistance was obtained from REF. 97.

There are two main reasons that make the prediction of antibiotic resistance based on the rate of acquisition of mutations of limited use. First, the methods that are currently used for assessing the probability of emergence of antibiotic resistance by mutation do not typically consider bacterial population sizes and within-patient dynamics, and they thereby overemphasize the importance of mutation rates in the emergence of antibiotic resistance genes. Indeed, in vitro mutation rates that were determined for our current repertoire of antibiotics span at least six logs (from $\sim 10^{-11}$ to $10^{-5}$ per cell per generation) at lethal drug concentrations $(>\mathrm{MIC})^{16,74}$. Moreover, the rate by which resistance has appeared in clinical settings is not obviously correlated to the mutation rates that are observed in vitro (FIG. 3). This finding could imply that, for most pathogens, the probability of selection and fixation of a resistant mutant in a patient is not strongly limited by the rate of acquisition of a mutation. A reasonable explanation for this is that resistant mutants are generally present in the infecting bacterial population when treatment is initiated, and therefore the time necessary for a resistant mutant to appear is short.

The examples of mecillinam, fosfomycin and nitrofurantoin resistance in Escherichia coli provide an experimental illustration of this concept. These antibiotics are used for the treatment of uncomplicated urinary tract infections, and the rates of emergence of mutations that confer antibiotic resistance are very high $\left(10^{-7}\right.$ to $10^{-5}$ per cell per generation). Owing to this high number of mutations in different genes, and the resulting high mutational rate, one would assume that resistance to these antibiotics should rapidly emerge ${ }^{16}$. In urinary tract infections, the population sizes range from $10^{4}$ to $10^{8}$ bacteria per $\mathrm{ml}$ of urine, resulting in total population sizes approaching $10^{10}$ in the full bladder ${ }^{15}$. With such large population sizes and great size of mutational targets, the expected number of resistant mutants in the population might be as high as $10^{5}$ (population size $10^{10} \times$ mutation rate $10^{-5}$ ) when treatment is initiated, and mathematical modelling suggests that resistance would develop very often during treatment ${ }^{15,16,75,76}$. However, the use of these antibiotics is rarely associated with the development of antibiotic resistance during treatment. This can be fully explained by the fitness costs that are associated with the resistance mechanisms, the pharmacodynamic response and the dynamics of the bladder environment. Indeed, although resistant mutants continually appear at a high rate, the fitness cost that is associated with resistance reduces the growth rate of the bacteria below the threshold that is required for the pathogen to be maintained in the high flow-rate environment of the bladder. In other words, even though the resistant mutants are generated at high rates they cannot be enriched because they grow too slowly to remain in the bladder during antibiotic exposure owing to continuous urine production and normal urination patterns. A significant implication of this is that other parameters, that is, the fitness costs of resistance, the pharmacodynamic response and the infection dynamics, are more relevant for evaluating the risks of developing antibiotic resistance.

Another reason for why current assessments of resistance are limited is because the majority of resistance mechanisms that are observed in pathogens are conferred by HGT of pre-existing genes that mediate antibiotic resistance, and in vitro measurements of the rates of emergence of mutations cannot inform us of the antibiotic resistance that is linked to HGT. HGT enables distantly related organisms to exchange genetic material ${ }^{77}$ and can be mediated through three mechanisms: transduction, transformation and conjugation. The potential for a pathogen to acquire a resistance gene through HGT is dependent on the density and the genetic complexity of the bacterial community in which it resides and on the selective pressures that are present. For pathogens that typically inhabit high-density and high-complexity environments, such as the gastrointestinal tract, HGT is likely to be a significant contributor in the acquisition of genes that confer antibiotic resistance. Indeed, most of the clinically challenging antibiotic resistance genes that are found in common pathogens, such as $\beta$-lactam resistance in S. aureus and Enterobacteriaceae and vancomycin resistance in enterococci, are horizontally acquired. The genes that confer these resistance phenotypes have been successful in their spread across the world, as highlighted by the rapid global spread of a specific class of $\beta$-lactamases: the CTX-M-type extended spectrum $\beta$-lactamase (ESBL) gene family ${ }^{78}$. Environmental metagenomic studies have found that genes that confer antibiotic resistance are prevalent in all environments, which suggests that the evolution of new resistance genes is not limited by the availability of functional antibiotic resistance genes ${ }^{79-82}$. Instead, factors such as mobilization of the potential antibiotic resistance gene, the fitness cost of the gene and the ecological overlap (that is, sharing of niches) between the recipient and donor environments seem to be important for the successful spread of horizontally acquired antibiotic resistance genes ${ }^{82,83}$. 


\section{A new preclinical paradigm}

As outlined above, the methods that are used for the preclinical assessment of the potential to evolve antibiotic resistance to new antibiotics are not adequate, as they largely disregard the role of HGT in the acquisition of antibiotic resistance and place too much emphasis on the rates of antibiotic resistance acquisition that are determined in vitro. Consequently, our decisions during drug development may be guided by clinically irrelevant parameters. Indeed, mecillinam, a broad-spectrum penicillin drug against which the frequency of antibiotic-resistant bacteria in clinical settings have remained low, would probably have been terminated at the preclinical stage on the basis of a very high rate of appearance of resistant mutants in laboratory test ${ }^{84}$. Accordingly, we should be cautious of ruling out antibiotic candidates based solely on mutation rates without further investigation of the properties of these resistant mutants and the relevant infection dynamics. In addition, we are currently not adequately assessing the potential for horizontal evolution of antibiotic resistance, even though this evolutionary process can render 'last-resort drugs', such as the carbapenems, ineffective.

Below, we propose how the experimental approaches that are used to assess both the vertical and horizontal evolution of resistance can be improved to make better-informed preclinical decisions for continuing or discontinuing drug development.

\section{Assessing vertical evolution of resistance.}

Several different factors influence the rate of emergence, spread and maintenance of any resistance gene, and a key question is which factors should be included in risk prediction. It is clear that mutation rates that are measured in vitro are poor predictors of clinical evolution of resistance - in particular for drugs that are associated with high rates of emergence of mutations. Furthermore, even though selective pressures, co-selection and epistatic interactions may markedly influence how resistance evolves, they are difficult to assess in antibiotic resistance prediction studies during preclinical development, as it is largely unknown how strong a future selective pressure could be or which co-selection processes or epistatic interactions might be important when a new antibiotic is introduced. Instead, we propose that the infection dynamics and mutation fitness costs in antibiotic-resistant bacteria should be considered to increase the relevance of risk predictions, simply because they are key parameters in determining rates of evolution of resistance, and there are experimental tools with which to measure them. Mutants that have a high fitness cost are less likely to get fixed (that is, become the dominant type in the population) in a competitive environment, despite conferring high levels of antibiotic resistance. By contrast, mutations that confer low levels of antibiotic resistance can be fixed in the population if their fitness cost is low. Furthermore, the rate and efficiency of the evolution of compensatory mutations, which could restore fitness without the loss of antibiotic resistance, are key parameters in determining the long-term fate of resistant mutants ${ }^{21}$. A repertoire of methods is available that enables fitness costs and the process of evolution of compensatory mutations to be analysed under several different in vitro and in vivo conditions, both in the absence and presence of an antibiotic pressure ${ }^{21,85}$. Similarly, the infection dynamics (that is, bacterial growth and killing rates, and population sizes) can be studied in relevant in vitro and animal infection models together with spontaneous mutation rates. Experimental methods for determining spontaneous mutation rates can be used to determine the probability that a resistant mutant emerges and becomes the dominant type in bacterial population in a specific host environment. Implementation of these approaches could revive antibiotic candidates that were discarded during drug development owing to a high rate of emergence of resistance mutations in vitro $^{9,15,16}$.

\section{Assessing horizontal evolution of resistance.} Despite the emergence of clinically relevant antibiotic resistance being most commonly the result of horizontal acquisition of resistance genes, assessment of the risk of acquisition of genes that confer antibiotic resistance is rarely carried out for new candidate antibiotics. This is surprising given that the methodology that is required to carry out such testing already exists ${ }^{86}$. Several parameters that affect HGT need to be considered, including whether potential resistance genes exist in nature, the possibility for HGT that is determined by niche constraints, the rate of HGT and the fitness effects on the host following HGT (FIG. 4).

Several recent studies have shown that all environments, including those without human influence (for example, tundra and caves), contain genes that confer resistance to the majority of antibiotics that are currently in use $\mathrm{e}^{79,80}$. Similarly, it has been shown that several clinically relevant antibiotic resistance genes (for example, $c t x-m$, $q n r A$ and tet $X$, which confer resistance to $\beta$-lactams, quinolones, tetracyclines and aminoglycosides, respectively) originate from environmental bacteria and have recently transferred to pathogens ${ }^{87,88}$. Thus, the most important question during the risk assessment of a new antibiotic is whether genes that could potentially mediate resistance to antibiotics already exist in the resistome (that is, the pool of all resistance genes that are present in a certain environment), in particular in environments in which the relevant human pathogens and potential donor bacteria are likely to interact.

Using functional metagenomic selection, in which expression libraries of metagenomic DNA are transformed into a relevant host and screened for phenotypic resistance, it is possible to investigate whether a specific environment harbours potential resistance genes against a new candidate drug ${ }^{86}$ (FIG. 4a). This method can be expanded by using metagenomic libraries from different environments, with varying insert sizes and expression platforms. In addition, the screening can be carried out using different host bacteria and selection conditions, such as different growth media, growth conditions and selection strengths. By using these different screening methods, it is possible to reduce some of the biases that are associated with heterologous gene expression, such as differences in expression that are dependent on genetic background and/or the growth environment used for screening.

Although it is impractical to conduct metagenomic functional selections for all environments, we suggest that human microbiomes with which an infecting pathogen is likely to interact are investigated and screened. In addition, microbiomes that contain genetic material from diverse origins, such as those from waste water as well as the environments in which antibiotic producers are found, may be important to screen. Any identified antibiotic resistance gene represents a potential risk for the evolution and dissemination of clinically relevant antibiotic resistance ${ }^{89}$. Therefore, metagenomic HGT experiments also provide an opportunity to act as an early warning system for downstream surveillance studies. However, the magnitude of this risk is dependent on several factors ( $F I G .4 b-d)$, including ecological opportunity (contact between a potential donor bacterium and a recipient pathogen), in vivo HGT rates between the relevant species, the likelihood of fixation of the acquired DNA (which largely depends on the fitness costs of the transferred gene), and gene expression 
occurring at a sufficiently high level to confer clinically relevant antibiotic resistance to the pathogen. All of these parameters, except for ecological opportunity, can be experimentally determined to provide the basis for a rational risk assessment. Ecological opportunity can be partially assessed using bioinformatics approaches that compare metagenomic datasets from diverse environments to a selected database comprising the gene or genes that, when overexpressed, can cause resistance to antibiotics.

\section{Outlook}

On the basis of the knowledge we have today on the evolution of antibiotic resistance, we propose that it is time for an updated preclinical paradigm to assess the risk of resistance development in early preclinical development. This change is feasible and is not limited by methodology but rather by tradition. Although the preclinical assessments that have been suggested in this Opinion article represent an additional burden on antibiotic developers, with a

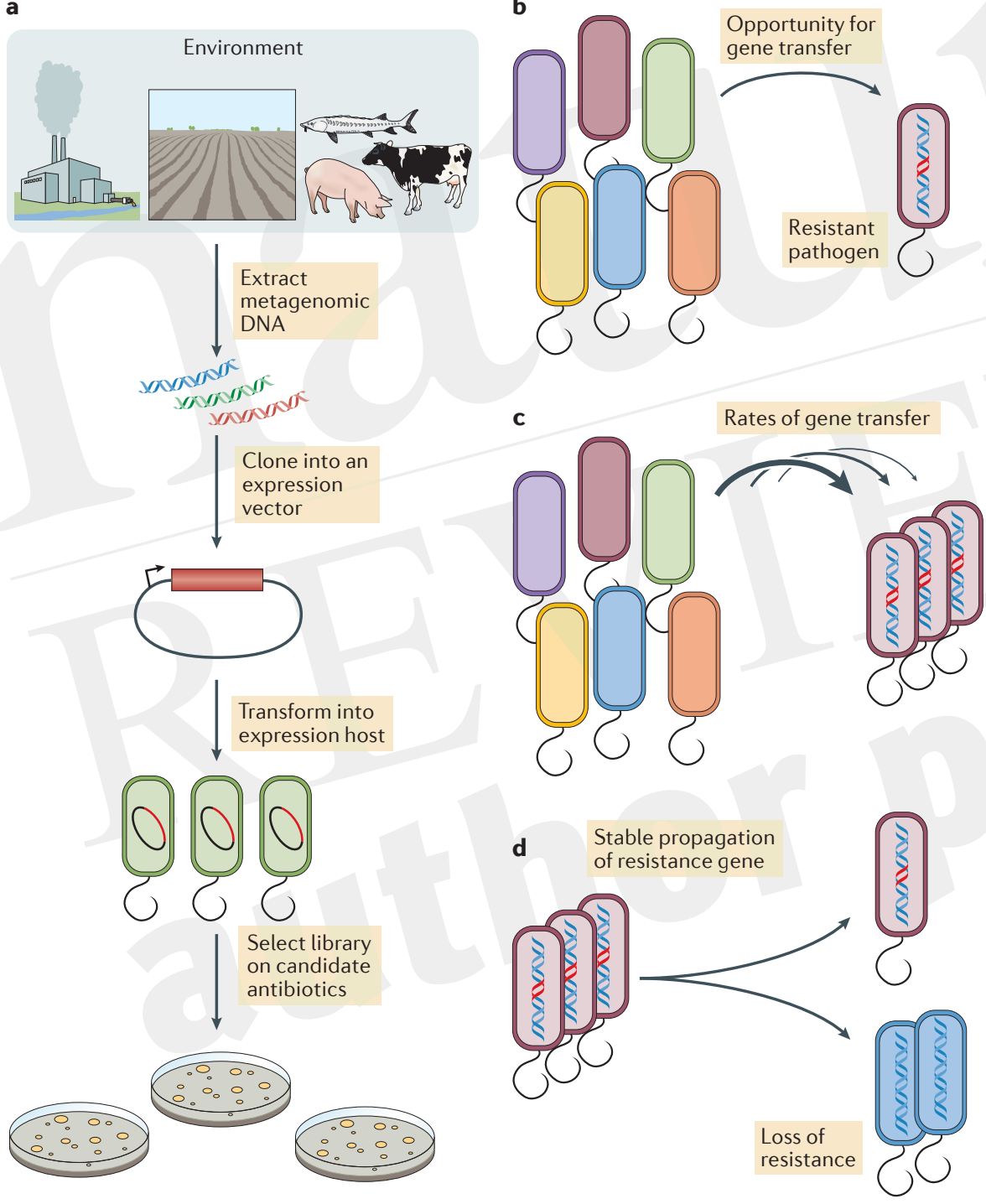

Figure 4 | Examining the potential of horizontal gene transfer. The genetic reservoirs across different natural and anthropogenic environments are likely to contain genes that can confer resistance to novel antibiotics. Functional metagenomic selection enables high-throughput screening to identify potential resistance genes. For high-throughput screening, after the extraction of DNA, the DNA library that contains potential resistance-mediating genes is cloned and transformed into suitable expression hosts and then selected using the candidate antibiotic (part a). Following the identification of potential resistance genes, it is necessary to assess (part b) the opportunity for gene transfer to the pathogen (that is, interactions between environmental reservoir and pathogen), the rate of gene transfer (part c) and the ability of the acquired resistance gene to be stably propagated in the pathogen (part d), as opposed to the possibility of being lost. Blue cells are sensitive bacteria, whereas red cells are resistant.

regards to time and cost, these assessments have the potential to add substantial value to antibiotic development programmes at the preclinical stage. Indeed, such an integrative approach to preclinical assessment of evolution of resistance should enable improved clinical predictions and help inform decisions during the drug development process.

Moreover, the proposed integrated framework may also re-activate shelved antibiotic development programmes that were once considered too prone to develop resistance based on traditional methods. Indeed, antibiotics with a high emergence of resistance mutations, as determined in vitro, may not be prone to develop antibiotic resistance in the clinic. As evidenced by the example of mecillinam, the fitness cost of antibiotic resistance mutations and infection dynamics substantially affect clinically relevant evolution of resistance and must be considered along with the characterization of the rate of acquisition of resistance mutations. Accordingly, we believe that the proposed change in preclinical characterization of antibiotic resistance evolution will improve the long-term efficiency of antibiotic drug development.

Morten O. A. Sommer is at AntibioTx A/S, Kemitorvet Lyngby DK-2800, Denmark; and at The Novo Nordisk Foundation Center for Biosustainability, Technical University of Denmark, Lyngby DK-2800, Denmark.

Christian Munck is at The Novo Nordisk Foundation Center for Biosustainability, Technical University of Denmark, Lyngby DK-2800, Denmark.

Rasmus Vendler Toft-Kehler is at AntibioTx A/S, Kemitorvet, Lyngby DK-2800, Denmark.

Dan I. Andersson is at Uppsala University, Department of Medical Biochemistry and Microbiology, Husargatan 3, SE-751 23 Uppsala, Sweden.

Correspondence to D.I.A. Dan.Andersson@imbim.uu.se

doi: 10.1038/nrmicro.2017.75 Published online DD Mon 2017

1. [No authors listed.] Tackling drug-resistant infections globally: final report and recommendations (review on antimicrobial resistance, 2016). AMR Review https:// amr-review.org/sites/default/files/160525_Final\%20 paper_with\%20cover.pdf (2016).

2. Gilmore, M. S., Lebreton, F. \& van Schaik, W. Genomic transition of enterococci from gut commensals to leading causes of multidrug-resistant hospital infection in the antibiotic era. Curr. Opin. Microbiol. 16, 10-16 (2013).

3. zur Wiesch, P. A., Kouyos, R., Engelstädter, J., Regoes, R. R. \& Bonhoeffer, S. Population biological principles of drug-resistance evolution in infectious diseases. Lancet Infect. Dis. 11, 236-247 (2011).

4. Smith, M. R. \& Wood, W. B. An experimental analysis of the curative action of penicillin in acute bacterial infections. III. The effect of suppuration upon the antibacterial action of the drug. J. Exp. Med. 103, 509-522 (1956)

5. Palaci, M. et al. Cavitary disease and quantitative sputum bacillary load in cases of pulmonary tuberculosis. J. Clin. Microbiol. 45, 4064-4066 (2007). 
6. Feldman, W. E. Concentrations of bacteria in cerebrospinal fluid of patients with bacterial meningitis. J. Pediatr. 88, 549-552 (1976).

7. Canetti, G. Present aspects of bacterial resistance in tuberculosis. Am. Rev. Respir. Dis. 92, 687-703 (1965).

8. Canetti, G. Dynamic aspects of the pathology and bacteriology of tuberculous lesions. Am. Rev. Tuberc. 74, 13-21 (1956)

9. Foster, P. L. Methods for determining spontaneous mutation rates. Methods Enzymol. 409, 195-213 (2006).

10. Drake, J. W., Charlesworth, B., Charlesworth, D. \& Crow, J. F. Rates of spontaneous mutation. Genetics 148, 1667-1686 (1998)

11. Lynch, M. Evolution of the mutation rate. Trends Genet 26, 345-352 (2010)

12. Tubulekas, I., Buckingham, R. H. \& Hughes, D. Mutant ribosomes can generate dominant kirromycin resistance. J. Bacteriol. 173, 3635-3643 (1991).

13. Lofton, H., Pränting, M., Thulin, E. \& Andersson, D. I. Mechanisms and fitness costs of resistance to antimicrobial peptides LL-37, CNY100HL and wheat germ histones. PLoS ONE 8, e68875 (2013).

14. Gullberg, E. et al. Selection of resistant bacteria at very low antibiotic concentrations. PLoS Pathog. 7 , e 1002158 (2011).

15. Nilsson, A. I., Berg, O. G., Aspevall, O., Kahlmeter, G, $\&$ Andersson, D. I. Biological costs and mechanisms of fosfomycin resistance in Escherichia coli. Antimicrob. Agents Chemother. 47, 2850-2858 (2003).

16. Thulin, E., Sundqvist, M. \& Andersson, D. I. Amdinocillin (mecillinam) resistance mutations in clinical isolates and laboratory-selected mutants of Escherichia coli. Antimicrob. Agents Chemother. $\mathbf{5 9}$ 1718-1727 (2015).

17. Drusano, G. L., Louie, A., MacGowan, A. \& Hope, W Suppression of emergence of resistance in pathogenic bacteria: keeping our powder dry, part 1 .

Antimicrob. Agents Chemother. 60, 1183-1193 (2015).

18. Drusano, G. L., Hope, W., MacGowan, A. \& Louie, A Suppression of emergence of resistance in pathogenic bacteria: keeping our powder dry, part 2. Antimicrob. Agents Chemother. 60, 1194-1201 (2015).

19. Chancey, S. T., Zähner, D. \& Stephens, D. S. Acquired inducible antimicrobial resistance in Gram-positive bacteria. Future Microbiol. 7, 959-978 (2012).

20. Chevereau, G. et al. Quantifying the determinants of evolutionary dynamics leading to drug resistance. PLOS Biol. 13, e1002299 (2015)

21. Andersson, D. I. \& Hughes, D. Antibiotic resistance and its cost: is it possible to reverse resistance? Nat. Rev. Microbiol. 8, 260-271 (2010).

22. Andersson, D. I. \& Levin, B. R. The biological cost of antibiotic resistance. Curr. Opin. Microbiol. 2, 489493 (1999).

23. Wiser, M. J., Ribeck, N. \& Lenski, R. E. Long-term dynamics of adaptation in asexual populations. Science 342, 1364-1367 (2013).

24. Hughes, D. \& Andersson, D. I. Evolutionary consequences of drug resistance: shared principles across diverse targets and organisms. Nat. Rev. Genet 16, 459-471 (2015).

25. Brandis, G., Pietsch, F., Alemayehu, R. \& Hughes, D. Comprehensive phenotypic characterization of rifampicin resistance mutations in Salmonella provides insight into the evolution of resistance in Mycobacterium tuberculosis. J. Antimicrob. Chemother. 70, 680-685 (2015).

26. O'Neill, A. J., Huovinen, T., Fishwick, C. W. G. \& Chopra, I. Molecular genetic and structural modeling studies of Staphylococcus aureus RNA polymerase and the fitness of rifampin resistance genotypes in relation to clinical prevalence. Antimicrob. Agents Chemother. 50, 298-309 (2006)

27. Bottger, E. C., Springer, B., Pletschette, M. \& Sander, P. Fitness of antibiotic-resistant microorganisms and compensatory mutations. Nat. Med. 4, 1343-1344 (1998).

28. Sander, P. et al. Fitness cost of chromosomal drug resistance-conferring mutations. Antimicrob. Agents Chemother. 46, 1204-1211 (2002).

29. Shcherbakov, D. et al. Directed mutagenesis of Mycobacterium smegmatis 16S rRNA to reconstruct the in vivo evolution of aminoglycoside resistance in Mycobacterium tuberculosis. Mol. Microbiol. 77 830-840 (2010)

30. Foucault, M.-L., Depardieu, F., Courvalin, P. \& GrillotCourvalin, $C$. Inducible expression eliminates the fitness cost of vancomycin resistance in enterococci.
Proc. Natl Acad. Sci. USA 107, 16964-16969 (2010)

31. Andersson, D. I. \& Hughes, D. Microbiological effects of sublethal levels of antibiotics. Nat. Rev. Microbiol. 12, 465-478 (2014).

32. Gullberg, E., Albrecht, L. M., Karlsson, C., Sandegren, L. \& Andersson, D. I. Selection of a multidrug resistance plasmid by sublethal levels of antibiotics and heavy metals. mBio 5, e01918-14 (2014).

33. Oz, T. et al. Strength of selection pressure is an important parameter contributing to the complexity of antibiotic resistance evolution. Mol. Biol. Evol. 31 2387-2401 (2014).

34. De Visser, J. A. G. M. \& Krug, J. Empirical fitness landscapes and the predictability of evolution. Nat. Rev. Genet. 15, 480-490 (2014).

35. Kondrashov, D. A. \& Kondrashov, F. A. Topological features of rugged fitness landscapes in sequence space. Trends Genet. 31, 24-33 (2015).

36. Brandis, G. \& Hughes, D. Genetic characterization of compensatory evolution in strains carrying $r p o B$ Ser531Leu, the rifampicin resistance mutation most frequently found in clinical isolates. J. Antimicrob. Chemother. 68, 2493-2497 (2013).

37. Brandis, G., Wrande, M., Liljas, L. \& Hughes, D. Fitness-compensatory mutations in rifampicinresistant RNA polymerase. Mol. Microbiol. 85 142-151 (2012).

38. Lannergård, J. et al. Genetic complexity of fusidic acid resistant small colony variants (SCV) in Staphylococcus aureus. PLOS ONE 6, e28366 (2011).

39. Marcusson, L. L., Frimodt-Møller, N. \& Hughes, D. Interplay in the selection of fluoroquinolone resistance and bacterial fitness. PLoS Pathog. 5, e 1000541 (2009).

40. Schrag, S. J., Perrot, V. \& Levin, B. R. Adaptation to the fitness costs of antibiotic resistance in Escherichia coli. Proc. Biol. Sci. 264, 1287-1291 (1997).

41. Angst, D. C. \& Hall, A. R. The cost of antibiotic resistance depends on evolutionary history in Escherichia coli. BMC Evol. Biol. 13, 163 (2013).

42. Komp Lindgren, P., Marcusson, L. L., Sandvang, D., Frimodt-Møller, N. \& Hughes, D. Biological cost of single and multiple norfloxacin resistance mutations in Escherichia coli implicated in urinary tract infections. Antimicrob. Agents Chemother. 49, 2343-2351 (2005).

43. Trindade, S. et al. Positive epistasis drives the acquisition of multidrug resistance. PLoS Genet. 5 e1000578 (2009)

44. Björkman, J., Samuelsson, P., Andersson, D. I. \& Hughes, D. Novel ribosomal mutations affecting translational accuracy, antibiotic resistance and virulence of Salmonella typhimurium. Mol. Microbiol. 31, 53-58 (1999).

45. Hall, A. R. \& MacLean, R. C. Epistasis buffers the fitness effects of rifampicin-resistance mutations in Pseudomonas aeruginosa. Evolution 70, 1161-116 (2016)

46. Rozen, D. E., McGee, L., Levin, B. R. \& Klugman, K. P. Fitness costs of fluoroquinolone resistance in Streptococcus pneumoniae. Antimicrob. Agents Chemother. 51, 412-416 (2007).

47. Vogwill, T., Kojadinovic, M. \& MacLean, R. C. Epistasis between antibiotic resistance mutations and genetic background shape the fitness effect of resistance across species of Pseudomonas. Proc. Biol. Sci. 283 20160151 (2016)

48. Vogwill, T. \& MacLean, R. C. The genetic basis of the fitness costs of antimicrobial resistance: a metaanalysis approach. Evol. Appl. 8, 284-295 (2015)

49. Johanson, U., Ævarsson, A., Liljas, A. \& Hughes, D. The dynamic structure of EF-G studied by fusidic acid resistance and internal revertants. J. Mol. Biol. 258 420-432 (1996).

50. Nagaev, I., Bjorkman, J., Andersson, D. I. \& Hughes, D. Biological cost and compensatory evolution in fusidic acid-resistant Staphylococcus aureus. Mol. Microbiol. 40, 433-439 (2001).

51. Salverda, M. L. M. et al. Initial mutations direct alternative pathways of protein evolution. PLoS Genet. 7, e1001321 (2011)

52. Weinreich, D. M. Delaney, N. F., Depristo, M. A. \& Hartl, D. L. Darwinian evolution can follow only very few mutational paths to fitter proteins. Science 312 111-114 (2006).

53. Dahlberg, C. \& Chao, L. Amelioration of the cost of conjugative plasmid carriage in Eschericha coli K12. Genetics 165, 1641-1649 (2003).
54. Loftie-Eaton, W. et al. Evolutionary paths that expand plasmid host-range: implications for spread of antibiotic resistance. Mol. Biol. Evol. 33, 885-897 (2016).

55. San Millan, A., Heilbron, K. \& MacLean, R. C. Positive epistasis between co-infecting plasmids promotes plasmid survival in bacterial populations. ISME J. 8 , 601-612 (2014)

56. San Millan, A. et al. Positive selection and compensatory adaptation interact to stabilize nontransmissible plasmids. Nat. Commun. 5, 5208-5211 (2014)

57. Silva, R. F. et al. Pervasive sign epistasis between conjugative plasmids and drug-resistance chromosomal mutations. PLoS Genet. 7, e1002181 (2011).

58. Porse, A., Schønning, K., Munck, C. ¿ Sommer, M. O. A. Survival and evolution of a large multidrug resistance plasmid in new clinical bacterial hosts. Mol. Biol. Evol. 33, 2860-2873 (2016).

59. Alekshun, M. N. \& Levy, S. B. Molecular mechanisms of antibacterial multidrug resistance. Cell 128 , 1037-1050 (2007)

60. Palmer, A. C. \& Kishony, R. Understanding, predicting and manipulating the genotypic evolution of antibiotic resistance. Nat. Rev. Genet. 14, 243248 (2013).

61. Garcia, L. G. et al. Antibiotic activity against smallcolony variants of Staphylococcus aureus: review of in vitro, animal and clinical data. J. Antimicrob. Chemother. 68, 1455-1464 (2013).

62. Munck, C., Gumpert, H. K., Wallin, A. I. N. Wang, H. H. \& Sommer, M. O. A. Prediction of resistance development against drug combinations by collateral responses to component drugs. Sci. Trans Med. 6, 262ra156 (2014).

63. Imamovic, L. \& Sommer, M. O. A. Use of collateral sensitivity networks to design drug cycling protocols that avoid resistance development. Sci. Transl Med. 5 , 204ra132 (2013)

64. Kim, S., Lieberman, T. D. \& Kishony, R. Alternating antibiotic treatments constrain evolutionary paths to multidrug resistance. Proc. Natl Acad. Sci. USA 111, 14494-14499 (2014).

65. Lázár, V. et al. Genome-wide analysis captures the determinants of the antibiotic cross-resistance interaction network. Nat. Commun. 5, 4352 (2014).

66. Pena-Miller, R. et al. When the most potent combination of antibiotics selects for the greatest bacterial load: the smile-frown transition. PLOS Biol. 11, e 1001540 (2013).

67. Périchon, B. \& Courvalin, P. Synergism between $\beta$-lactams and glycopeptides against VanA-type methicillin-resistant Staphylococcus aureus and heterologous expression of the vanA operon. Antimicrob. Agents Chemother. 50, 3622-3630 (2006).

68. Brolund, A. \& Sandegren, L. Characterization of ESBL disseminating plasmids. Infect. Dis. (Lond.) 48, 18-25 (2016).

69. Mathers, A. J., Peirano, G. \& Pitout, J. D. D. The role of epidemic resistance plasmids and international high-risk clones in the spread of multidrug-resistant Enterobacteriaceae. Clin. Microbiol. Rev. 28 565-591 (2015)

70. Molton, J. S., Tambyah, P. A., Ang, B. S. P., Ling, M. L. $\&$ Fisher, D. A. The global spread of healthcareassociated multidrug-resistant bacteria: a perspective from Asia. Clin. Infect. Dis. 56, 1310-1318 (2013).

71. Bean, D. C., Livermore, D. M., Papa, I. \& Hall, L. M. C. Resistance among Escherichia coli to sulphonamides and other antimicrobials now little used in man. J. Antimicrob. Chemother. 56, 962-964 (2005).

72. Enne, V. I., Livermore, D. M., Stephens, P. \& Hall, L. M. Persistence of sulphonamide resistance in Escherichia coli in the UK despite national prescribing restriction. Lancet $357,1325-1328$ (2001).

73. Sundqvist, M. et al. Little evidence for reversibility of trimethoprim resistance after a drastic reduction in trimethoprim use. J. Antimicrob. Chemother. 65 350-360 (2010).

74. Locke, J. B., Hilgers, M. \& Shaw, K. J. Novel ribosomal mutations in Staphylococcus aureus strains identified through selection with the oxazolidinones linezolid and torezolid (TR-700). Antimicrob. Agents Chemother. 53, 5265-5274 (2009).

75. Gordon, D. M. \& Riley, M. A. A theoretical and experimental analysis of bacterial growth in the bladder. Mol. Microbiol. 6, 555-562 (1992). 
76. Sandegren, L., Lindqvist, A., Kahlmeter, G. \& Andersson, D. I. Nitrofurantoin resistance mechanism and fitness cost in Escherichia coli. J. Antimicrob. Chemother. 62, 495-503 (2008)

77. Thomas, C. M. \& Nielsen, K. M. Mechanisms of and barriers to, horizontal gene transfer between bacteria. Nat. Rev. Microbiol. 3, 711-721 (2005).

78. Naseer, U. \& Sundsfjord, A. The CTX-M conundrum: dissemination of plasmids and Escherichia coli clones. Microb. Drug Resist. 17 83-97 (2011).

79. Allen, H. K., Moe, L. A., Rodbumrer, J., Gaarder, A. ¿ Handelsman, J. Functional metagenomics reveals diverse $\beta$-lactamases in a remote Alaskan soil. ISME J. 3, 243-251 (2008).

80. D'Costa, V. M. et al. Antibiotic resistance is ancient. Nature 477, 457-461 (2011).

81. Sommer, M. O. A., Dantas, G. \& Church, G. M. Functional characterization of the antibiotic resistance reservoir in the human microflora. Science 325, 1128-1131 (2009).

82. Munck, C. et al. Limited dissemination of the wastewater treatment plant core resistome. Nat. Commun. 6, 8452 (2015).

83. Forsberg, K. J. et al. Bacterial phylogeny structures soil resistomes across habitats. Nature 509, 612-616 (2014).

84. Kahlmeter, G. \& Poulsen, H. O. Antimicrobial susceptibility of Escherichia coli from communityacquired urinary tract infections in Europe: the ECO.SENS study revisited. Int. J. Antimicrob. Agents 39, 45-51 (2012).
85. Huseby, D. L. et al. Mutation supply and relative fitness shape the genotypes of ciprofloxacin-resistant Escherichia coli. Mol. Biol. Evol. 34, 1029-1039 (2017).

86. Moore, A. M., Munck, C., Sommer, M. O. A. \& Dantas, G. Functional metagenomic investigations of the human intestinal microbiota. Front. Microbiol. 2 188 (2011)

87. Dantas, G. \& Sommer, M. O. Context matters - the complex interplay between resistome genotypes and resistance phenotypes. Curr. Opin. Microbiol. 15 577-582 (2012)

88. Yoon, E.-J. et al. Origin in Acinetobacter gyllenbergii and dissemination of aminoglycoside-modifying enzyme AAC ( $\left.6^{\prime}\right)-$ Ih. J. Antimicrob. Chemother. 71 , 601-606 (2016).

89. Martínez, J. L., Coque, T. M. \& Baquero, F. Prioritizing risks of antibiotic resistance genes in all metagenomes. Nat. Rev. Microbiol. 13, 396-396 (2015).

90. Jaffé, A., Chabbert, Y. A. \& Derlot, E. Selection and characterization of $\beta$-lactam-resistant Escherichia coli K-12 mutants. Antimicrob. Agents Chemother. 23 , 622-625 (1983).

91. George, A. M. \& Levy, S. B. Amplifiable resistance to tetracycline, chloramphenicol, and other antibiotics in Escherichia coli: involvement of a non-plasmiddetermined efflux of tetracycline. J. Bacteriol. 155, 531-540 (1983).

92. Heisig, P. \& Tschorny, R. Characterization of fluoroquinolone-resistant mutants of escherichia coli selected in vitro. Antimicrob. Agents Chemother. 38 1284-1291 (1994).
93. Buckel, P. Buchberger, A., Böck, A. \& Wittmann, H. C. Alteration of ribosomal protein L6 in mutants of Escherichia coli resistant to gentamicin. Mol. Gen. Genet. 158, 47-54 (1977).

94. Adler, M., Anjum, M., Andersson, D. I. \& Sandegren, L. Influence of acquired $\beta$-lactamases on the evolution of spontaneous carbapenem resistance in Escherichia coli. J. Antimicrob. Chemother. 68, 51-59 (2013).

95. Oakberg, E. F. \& Luria, S. W. Mutations to sulfonamide resistance in Staphylococcus aureus. Genetics 32 249-261 (1947).

96. Zurenko, G. E. et al. In vitro activities of U-100592 and U-100766, novel oxazolidinone antibacterial agents. Antimicrob. Agents Chemother. 40, 839-845 (1996).

97. Lewis, K. Platforms for antibiotic discovery. Nat. Rev. Drug. Discov. 12, 371-387 (2013).

\section{Acknowledgements}

Work in the authors' laboratory was supported by grants from the Swedish Research Council (to D.I.A.), and the Novo Nordisk Foundation, the Lundbeck Foundation and the Danish Free Research Council (to M.O.A.S. and C.M.).

\section{Competing interests statement}

The authors declare competing interests: see Web version for details.

Publisher's note

Springer Nature remains neutral with regard to jurisdictional claims in published maps and institutional affiliations.

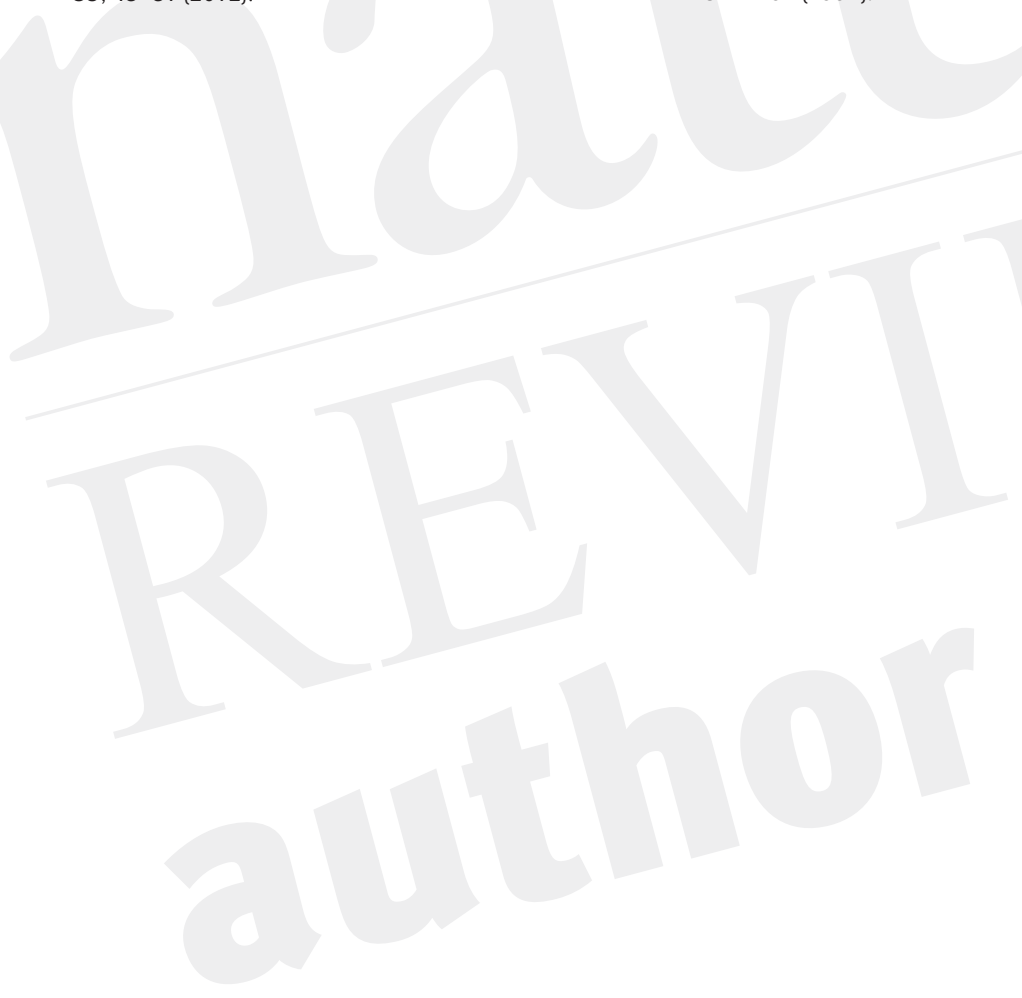


Author biographies

Morten Sommer is a professor at the Novo Nordisk Foundation Center for Biosustainability at the Technical University of Denmark. His work focuses on understanding and harnessing evolutionary processes and biological diversity. Genomeand metagenome-wide screens and perturbations are deployed in conjunction with synthetic biology tools to understand the evolution and phenotypic stability of biosynthetic processes, drug resistance and complex microbial communities. He received a Ph.D. in biophysics from Harvard University, Cambridge, Massachusetts, USA.

Christian Munck is a postdoctoral scientist in the laboratory of Harris Wang at Columbia University Medical Center, New York, USA. He received his Ph.D. from the Technical University of Denmark. His research interests centre on the evolution and dissemination of antibiotic resistance genes and bacterial adaptation to new environments.

Rasmus Toft-Kehler is the Co-founder of AntibioTx A/S and Clinical-Microbiomics A/S and holds an executive degree in entrepreneurial leadership from Harvard Business School, Boston, Massachusetts, USA, and a degree in finance and business administration from New York University, USA, and Copenhagen Business School, Denmark.

Dan I. Andersson is a professor of medical bacteriology at Uppsala University, Sweden. He has previously worked at University of Utah, Salt Lake City, USA, and the Swedish Institute for Infectious Disease Control, Solna. He uses genetics and experimental evolution to study genome stability and variability, development of antibiotic resistance and evolution of new genes.

\section{Competing interests statement}

The authors declare the following competing interests: M.O.A.S. and R.T.K. are shareholders in AntibioTx; CM declares no competing interests; D.I.A. is a consultant for Prebona and Bactiguard.

\section{Subject terms}

Biological sciences / Microbiology / Antimicrobials / Antimicrobial resistance

[URI /631/326/22/1434]

Biological sciences / Microbiology / Bacteria / Bacterial physiology / Antibacterial drug resistance

[URI /631/326/41/1969/2038]

Biological sciences / Microbiology / Bacteria / Bacterial evolution [URI /631/326/41/2529]

Biological sciences / Microbiology / Microbial genetics / Bacterial genetics

[URI /631/326/325/2482]

Biological sciences / Microbiology / Microbial communities /

Clinical microbiology

[URI /631/326/2565/107]

Biological sciences / Microbiology / Antimicrobials / Antibiotics

[URI /631/326/22/1290]

Biological sciences / Microbiology / Policy and public health in microbiology

[URI /631/326/1762]

\section{ToC blurb}

Prediction of antibiotic resistance: time for a new preclinical paradigm?

Morten O. A. Sommer, Christian Munck, Rasmus

Vendler Toft-Kehler and Dan I. Andersson

Risk assessment for the development of antibiotic resistance against a new drug candidate is of paramount importance in preclinical development. In this Opinion article, Sommer et al. propose a new preclinical paradigm for the prediction of antibiotic resistance.

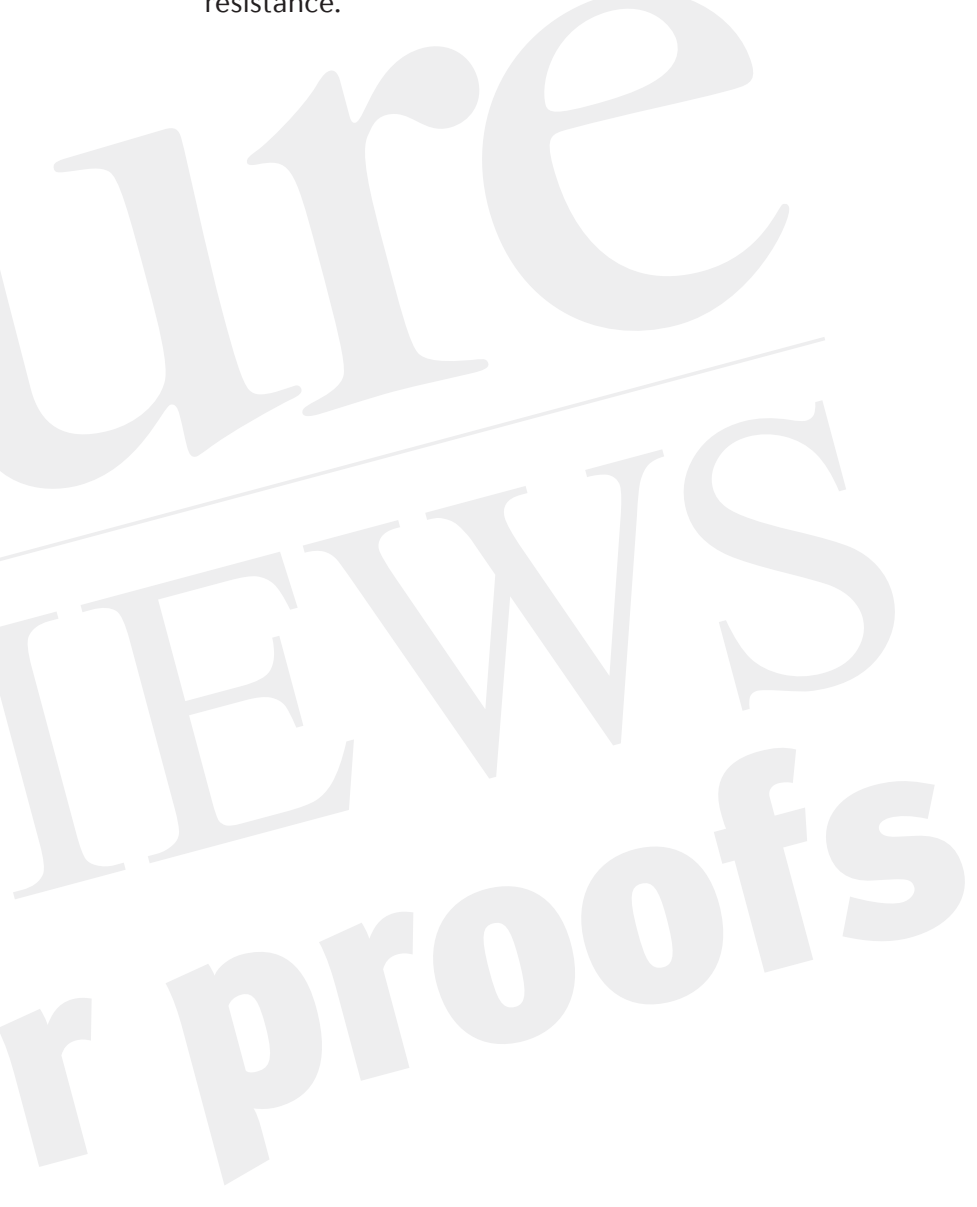

